\title{
Effects of ATRA on diabetic rats with renal ischemia-reperfusion injury ${ }^{1}$
}

\author{
Zeng Cheng'* (D) , Sun Qian" ${ }^{\prime *}(\mathbb{D})$, Meng Qingtao"l' (D) , Xia Zhongyuan"' (D) , Xiao Yeda"
}

' MM, Department of Anesthesiology, People's Hospital of Wuhan University, Wuhan, Hubei, China. Conception and design of the study, analysis and interpretation of data, manuscript preparation.

" PhD, Department of Anesthesiology, People's Hospital of Wuhan University, Wuhan, Hubei, China. Acquisition and analysis of data.

I'IPhD, Department of Anesthesiology, People's Hospital of Wuhan University, Wuhan, Hubei, China. Conception and design of the study, final approval.

*The authors contributed equally to this work

\begin{abstract}
Purpose: To explore the role of all-trans retinoic acid (ATRA) in renal ischemia/reperfusion injury of diabetic rats.

Methods: Sixty adult male rats were randomly divided into 6 groups, including sham group (S group), ischemia-reperfusion group (I/R group), ischemia-reperfusion+ATRA group (A group), diabetic group (D group), diabetic ischemia-reperfusion group (DI/R group), diabetic ischemia-reperfusion +ATRA group (DA group). The levels of creatinine $(\mathrm{Cr})$, cystatin $C(\mathrm{Cys}-\mathrm{C}$ ) and $\beta 2$-microglobulin ( $\beta 2-\mathrm{MG})$ were measured. Morphology of renal tissue was observed under light microscope.

Results: DJ-1, Nrf2, HO-1 and caspase-3 were detected by western blot. DJ-1, Nrf2, HO-1 and caspase-3 in I/R group, $\mathrm{D}$ group and $\mathrm{DI} / \mathrm{R}$ group was higher than that in $\mathrm{S}$ group. Compared with I/R group, Nrf2 and HO-1 in A group was decreased, but caspase-3 was increased. However, Nrf2 in DA group was higher than that in DI/R group, HO-1 and caspase-3 in DA group were lower than that in DI/R group. Compared with group $S, C r$, Cys- $C$ and $\beta 2-M G$ in I/R group, A group, D group, and DI/R group were higher. Whereas the levels of $\mathrm{Cr}$, Cys- $C, \beta 2-\mathrm{MG}$ and renal injury score in $\mathrm{DA}$ group were lower than those in DI/R group.
\end{abstract}

Conclusion: ATRA has a protective effect on renal ischemia-reperfusion injury in diabetic rats, maybe relating to DJ/Nrf2 pathway.

Key words: ATRA. Diabetes Mellitus. Ischemia. Reperfusion Injury. Rats. 


\section{- Introduction}

Diabetes mellitus, as a chronic metabolic disorder, is characterized by an abnormal insulin secretion and action resulting from interaction of hereditary and environmental factors. As one of the common complications of diabetes, diabetic nephropathy has an increasing gradually incidence. The pathogenesis of diabetic nephropathy is associated with the interaction of hyperglycemia, advanced glycation end-product, increased po-lyol pathway and oxidative stress ${ }^{1}$. During perioperative period, renal ischemia-reperfusion injury easily occurs in diabetic patients, which aggravates diabetic nephropathy. Due to surgery, especially the complex process of kidney surgery, stress, shock and prolonged stimulation resulted in renal insufficiency ${ }^{2}$.

All-trans retinoic acid (ATRA) is a potent derivative of vitamin A. Studies have shown that ATRA can effectively prevent the progression of diabetes in rats. Pancreatic $\beta$-cells, acinar and ductal cells gradually restore their normal appearance under ATRA treatment. What's more, insulin messenger RNA and serum indices almost normalize, which improves the histological changes of the pancreas and the serum indices in diabetic rats ${ }^{3,4}$. In addition, ATRA can affect the progression of diabetic nephropathy without causing any significant side effects and has a therapeutic effect on diabetic nephropathy ${ }^{5}$.

Sun et al. ${ }^{6}$ have demonstrated that DJ-1/Nrf2 pathway was involved in the pathogenesis of diabetic nephropathy in rats, which played a protective role on diabetic nephropathy. Nrf2/HO-1 pathway is one of the most important endogenous antioxidant pathway ${ }^{7,8}$. Upregulation of $\mathrm{Nrf} 2$ protein levels can protect the kidneys from I/R-associated oxidative damage ${ }^{9}$. Meanwhile, ATRA is an inhibitor of $\mathrm{Nrf2}^{10}$. This study aimed to investigate whether ATRA had a protective effect on renal ischemia- reperfusion in diabetic rats and whether it was related to DJ-1/Nrf2 pathway.

\section{- Materials}

Sixty adult SD male rats, weighing 200-220g, were purchased from the Animal Experimental Center of Wuhan University. Sixty SD Rats were fed 5 days after adaptation and were randomly assigned to six groups: sham group ( $S$ group), ischemia-reperfusion group (I/R group), ischemia-reperfusion +ATRA group (A group), diabetic group (D group), diabetic ischemia reperfusion group (DI/R group), diabetic ischemia reperfusion + ATRA group (DA group), every group $n=10$. ATRA was purchased from Sigma (R2625-100MG), and the rats were intraperitoneally injected with $1 \mathrm{mg} / \mathrm{mL}$ of ATRA at a dose of $1 \mathrm{~mL}$ per day.

\section{Diabetes model}

The rats were fasted overnight 12 hours before STZ injection. Then the rats received a single intraperitoneal injection of STZ (purchased from Sigma Chemical) at a dose of $60 \mathrm{mg} / \mathrm{kg}$ freshly dissolved in $0.1 \mathrm{M}$ citrate buffer ( $p h=4.5$ ). After 3 days of diabetes induction, tail vein blood glucose samples were collected and measured with OneTouch glucometer (Johnson \& Johnson, NJ). Rats with a FBG level above $16.7 \mathrm{mmol} / \mathrm{L}$ were considered diabetic and were selected ${ }^{11}$. Normal rats received an equivalent volume of citrate buffer only. The fasting blood glucose (FBG) level was monitored every week.

\section{Renal ischemia-reperfusion model ${ }^{12}$}

The rats were anesthetized with pentobarbital sodium $(50 \mathrm{mg} / \mathrm{kg}$ ) by intraperitoneal injection. The abdomen was opened, and the bilateral renal pedicles were occluded lasting for $30 \mathrm{~min}$, followed by $24 \mathrm{~h}$ of reperfusion.

\section{Measurement of serum $\mathrm{Cr}$, Cys-C and B2-MG}

Blood samples were collected by cardiac puncture, centrifuged at $3,000 \mathrm{rpm}$, for $10 \mathrm{~min}$ at $4^{\circ} \mathrm{C}$. Serum was separated and stored at $-20^{\circ} \mathrm{C}$. Levels of $\mathrm{Cr}$, Cys- $\mathrm{C}$ and $\beta 2-M G$ were measured using ELISA assay kits (cat nos. C011-1, E-EL-R0304c and E-EL-R1085c; Elabscience Biotechnology Co., Ltd., Wuhan, China) according to the manufacturer's instructions.

\section{Histological examination}

The left kidney was cut into sections and fixed in $4 \%$ formaldehyde, embedded in paraffin and sectioned into $4 \mu \mathrm{m}$ slices for light microscopy (original magnification, $\times 200$; Olympus BX50; Olympus Corporation), followed by staining with hematoxylin and eosin (HE). Histological assessment of tubular necrosis was semi-quantitatively determined using a method modified from McWhinnie et al. ${ }^{13}$ using the following scoring system: 0), normal histology; 1), tubular cell swelling, brush border loss and nuclear condensation, with up to one-third of the tubular profile exhibiting nuclear loss; 2), same as for score 1 , but more than one-third and less than two-thirds of the tubular profile displaying nuclear loss; and 3), same as for score 1, but more than two-thirds of the tubular profile exhibiting nuclear loss.

\section{Western blot analysis}

Western blot analysis was performed as previously described $^{14,15}$. Briefly, equal amounts of protein $(50 \mu \mathrm{g})$ 
were separated by $12 \%$ SDS-PAGE at $100 \mathrm{~V}$ for $3 \mathrm{~h}$. After electrophoresis, proteins were transferred onto polyvinylidenedifluoride membranes at $200 \mathrm{~mA}$ for 70 min. The membranes were incubated with the primary antibodies (against active DJ-1, Nrf2, HO-1, caspase-3). After washing three times in TBS-T, membranes were incubated with anti-rabbit immunoglobulin G conjugated to horseradish peroxidase at a dilution of 1: 2,000 in TBS-T containing $5 \%$ skimmed milk for $2 \mathrm{~h}$ at room temperature. The immunoreactive bands were visualized by enhanced chemiluminescence (PerkinElmer, Inc., Waltham, MA, USA) and captured on X-ray film. Blots were stained with an anti- $\beta$-actin antibody, and the protein levels were normalized with respect to $\beta$-actin band density.

\section{Statistical analysis}

All data are expressed as the mean \pm standard deviation (SD). Statistical analysis was performed using GraphPad Prism software version 7.0 (GraphPad Software, Inc., La Jolla, CA, USA). Analysis of variance
(ANOVA) test and Student's t test were used to evaluate statistical significance. The level of significance was set at $\mathrm{P}<0.05$ for all statistical tests.

\section{- Results}

\section{Renal histopathology}

Edema, tubular cell necrosis and cytoplasmic vacuoles were observed in histological specimens in I/R group, A group, D group and DI/R group, but were absent in $\mathrm{S}$ group. Compared with the renal histological evaluation score of kidneys obtained from $\mathrm{S}$ group, $I / R$ group and $D$ group exhibited a clearly increase in renal histologic evaluation score $(P<0.05)$. This increase was significantly enhanced in $I / R$ group and $D I / R$ group compared to I/R group and $D$ group individually respectively $(P<0.05)$. Histological alterations were obviously improved in specimens from DA group compared to DI/R group (Fig. $1, P<0.05$ ).

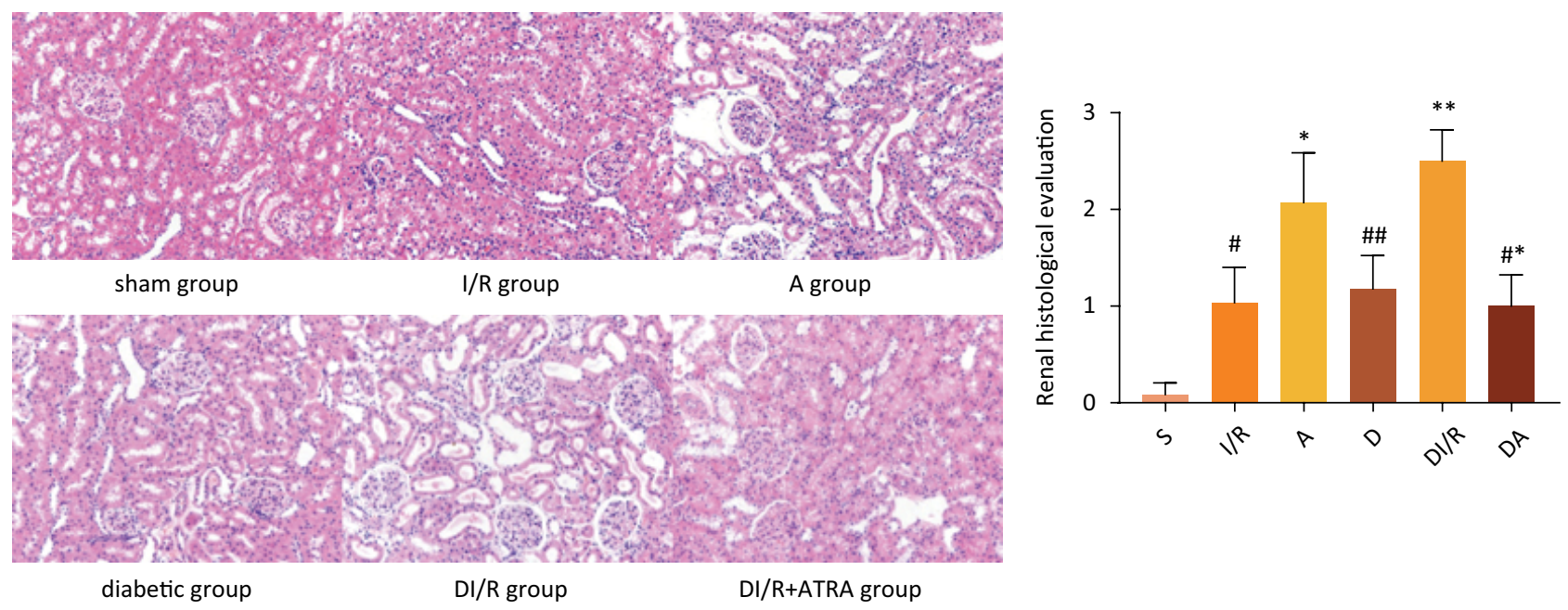

Figure 1 - Renal histological evaluation of kidney injury and quantitative injury scores (hematoxylin and eosin staining; magnification, $\times 200)$. Data are expressed as the mean \pm standard deviation $(n=10) .{ }^{\#} P<0.05$ vs. sham group, ${ }^{*} P<0.05$ vs. I/R group, ${ }^{\# \#} P<0.05$ vs. sham group, ${ }^{* *} P<0.05$ vs. D group, ${ }^{\#^{*}} P<0.05$ vs. DI/R group.

\section{Serum level of Cr, Cys-C and B2-MG}

The serum levels of $\mathrm{Cr}$, Cys-C and $\beta 2-\mathrm{MG}$ in $\mathrm{I} / \mathrm{R}$ group and $D$ group were significantly higher than in $S$ group $(P<0.05)$. And those blood indices were increased furtherly in A group and DI/R group compared to $\mathrm{I} / \mathrm{R}$ group and $D$ group individually $(P<0.05)$. Administration of ATRA visibly reduced the $\mathrm{Cr}$, Cys- $\mathrm{C}$ and $\beta 2-\mathrm{MG}$ levels compared with DI/R group rats (Fig. 2, $P<0.05$ ). 


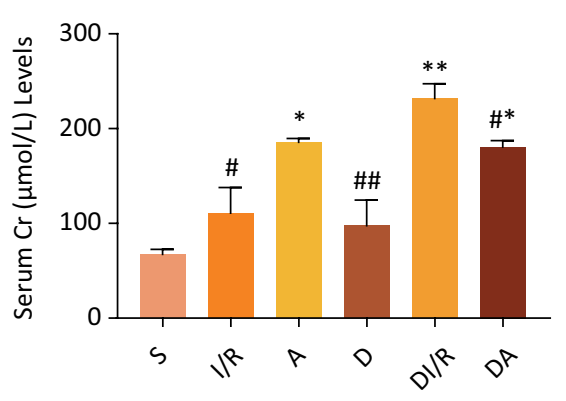

(1)

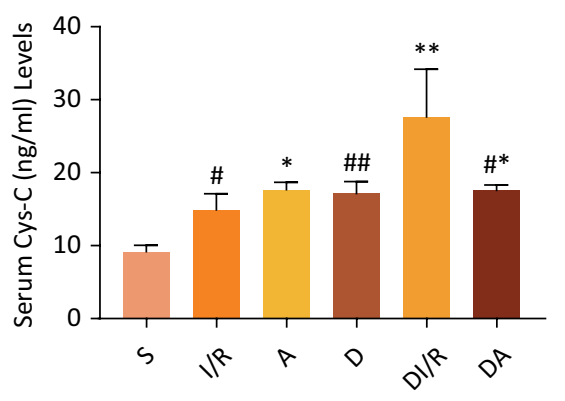

(2)

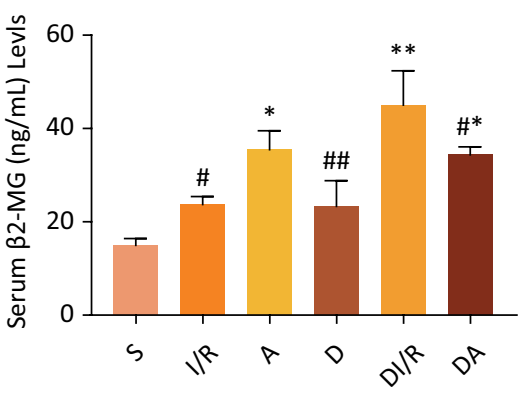

(3)

Figure 2 - Serum level of Cr, Cys-C and $\beta 2-M G$. (1) Cr:S group 67.27 $\pm 5.46 \mu \mathrm{mol}, \mathrm{I} / \mathrm{R}$ group: $111.00 \pm 26.97 \mu \mathrm{mol}, \mathrm{A}$ group: $185.5 \pm 4.20 \mu \mathrm{mol}, \mathrm{D}$ group: $97.56 \pm 27.07 \mu \mathrm{mol}, \mathrm{DI} / \mathrm{R}$ group: $231.84 \pm 15.57 \mu \mathrm{mol}, \mathrm{DA}$ group: $180.50 \pm 6.76 \mu \mathrm{mol}$; (2) Cys-C: $\mathrm{S}$ group: $9.18 \pm 0.88 \mathrm{ng}, \mathrm{l} / \mathrm{R}$ group: $14.92 \pm 2.19 \mathrm{ng}$, A group: $17.67 \pm 1.02 \mathrm{ng}, \mathrm{D}$ group: $17.14 \pm 1.64 \mathrm{ng}$, DI/R group: $27.66 \pm 6.53 \mathrm{ng}$, DA group: $17.57 \pm 0.74 \mathrm{ng}$; (3) $\beta 2-M G$ : S group: $14.99 \pm 1.41 \mathrm{ng}, \mathrm{l} / \mathrm{R}$ group: $23.73 \pm 1.68 \mathrm{ng}$, A group: $35.46 \pm 4.08 \mathrm{ng}, \mathrm{D}$ group: $23.32 \pm 5.48 \mathrm{ng}, \mathrm{DI} / \mathrm{R}$ group: $45.01 \pm 7.33 \mathrm{ng}$, DA group: $34.36 \pm 1.72 \mathrm{ng}$. Data are expressed as the mean \pm standard deviation (n=10). ${ }^{\# P<0.05}$ vs. sham group, ${ }^{*} P<0.05$ vs. I/R group, ${ }^{\#} P<0.05$ vs. sham group, ${ }^{* *} P<0.05$ vs. $D$ group, ${ }^{* *} P<0.05$ vs. $D I / R$ group.

The expression of DJ-1, Nrf2, $\mathrm{HO}-1$ and caspase-3 in renal tissues

Compared with sham group, the expression of DJ-1, Nrf2, HO-1 and caspase-3 in I/R group and D group were increased remarkably. And administration of ATRA significantly decreased the expression of Nrf2 and HO-1 in $A$ group contrasted with $\mathrm{I} / \mathrm{R}$ group. Moreover, the expression of DJ-1 and caspase-3 in A group strengthened much more than in I/R group. In DI/R group, the expression of DJ-1, Nrf2, HO-1 and caspase-3 also were enhanced in contrast with $D$ group. However, the expression of Nrf2 and $\mathrm{HO}-1$ in DA group was heightened compared with $\mathrm{DI} / \mathrm{R}$ group, and the caspase- 3 expression in DA group reduced perceptibly (Fig. $3, P<0.05$ ).

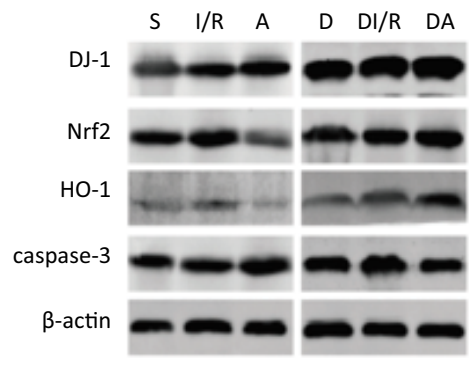

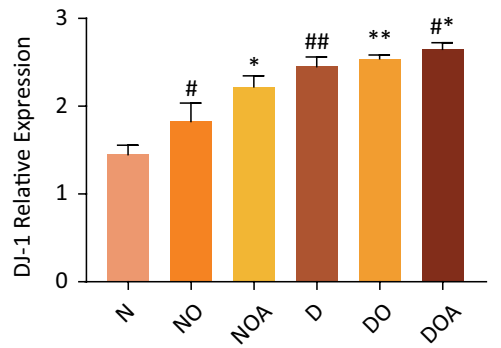

(1)

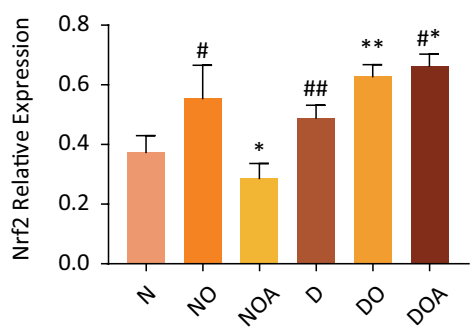

(2)

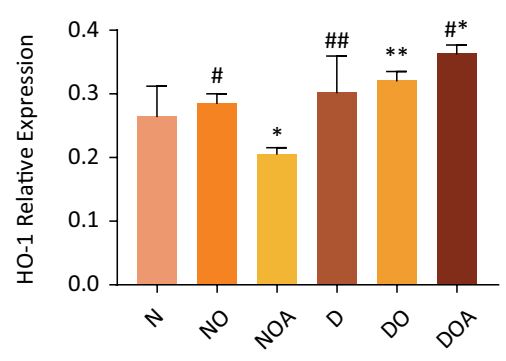

(3)

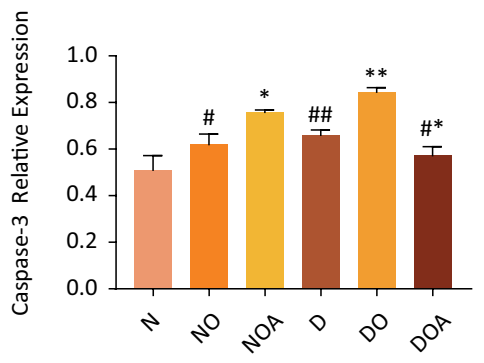

(4)

Figure 3 - The protein expression of DJ-1, Nrf2, HO-1 and caspase-3. As examined by western blotting, and proteins level DJ-1, Nrf2, HO-1 and caspase-3 in the renal tissue. Data are expressed as the mean \pm standard deviation ( $\mathrm{n}=10) .{ }^{\#} P<0.05$ vs. sham group, ${ }^{*} P<0.05$ vs. I/R group, ${ }^{\# \#} P<0.05$ vs. sham group, ${ }^{* *} P<0.05$ vs. D group, ${ }^{\# *} P<0.05$ vs. DI/R group. 


\section{- Discussion}

The present study found that ATRA inhibited the expression of Nrf2 leading to a decreasing of HO-1 in A group, but the expression of caspase-3, the serum levels of $\mathrm{Cr}$, Cys-C and $\beta 2-\mathrm{MG}$ and the renal histological evaluation score of kidneys were increased in $A$ group more than those in I/R group. It demonstrated that ATRA aggravated kidney damage caused by renal ischemiareperfusion. However, when ATRA was administrated in diabetic rats with ischemia-reperfusion, the expression of Nrf2 strengthened while the expression of caspase-3, the serum levels of $\mathrm{Cr}$, Cys-C and $\beta 2-\mathrm{MG}$ and the renal histological evaluation score of kidneys was decreased in comparison with DI/R group. Thus, ATRA not only improved the histological alterations and the blood indices, but also ameliorated kidney function and mitigated kidney damage.

ATRA exerts beneficial effects on nephropathy ${ }^{16-18}$. ATRA improves the histological changes of the pancreas of diabetic rats resulting in a significant decrease in blood glucose and total peroxides, elevated pancreatic insulin mRNA, and serum insulin ${ }^{4}$. ATRA shows to limit glomerular cell proliferation and kidney damage by reducing renal TGF- $\beta 1$ and TGF receptor II expression in nephropathy rat models ${ }^{16}$. ATRA reduces the incidence of T1DM by protecting islet $\beta$-cells, upregulating the levels of the pancreatic $\beta$-cell factor IL-4, reducing the cell toxicity-associated levels of IFN- $\gamma$ in $\beta$-cells, and maintaining the Th1/Th2 balance ${ }^{3}$. What's more, ATRA exhibits an anti-oxidant property by blocking lipid peroxidation in streptozotocin-induced diabetic rats ${ }^{19}$. It has been suggested that oxidative stress, as a key factor, is involved in the pathogenesis and progression of diabetic nephropathy (DN) ${ }^{20-22}$. DJ-1/Nrf2 pathway has been found to perform a protective effect on diabetic nephropathy ${ }^{6,23}$, which is one of the most important endogenous antioxidant pathway,8. In this study, the expression of DJ-1/Nrf2/HO-1 in DA group was upregulated, and histological alterations clearly improved edema, as tubular cell necrosis and cytoplasmic vacuoles almost disappeared. These data suggested that ATRA exerted protective effects in STZ-induced diabetic rats with renal ischemia-reperfusion injury.

In the renal ischemia-reperfusion rats model, increased oxidative stress also participates in the pathogenesis of renal I/R and deteriorates kidney injury ${ }^{24,25}$. Nrf2 is known as the "master regulator" of antioxidant/antiinflammatory/cytoprotective responses ${ }^{26-28}$. Under normal conditions, Nrf2 is retained in the cytoplasm, being bound to KEAP1, which targets it for ubiquination and proteosomal destruction ${ }^{27-29}$. In response to oxidative stress, critical KEAP1 cysteine residues are disrupted, Nrf2 is thus freed from ubiquination, and enhanced nuclear translocation results. In concert with a small Maf protein, and with export of the nuclear repressor Bach1, Nrf2 binds to antioxidant response elements (AREs) in the promoter regions of diverse cytoprotective genes. However, ATRA is an inhibitor of transcription factor $\mathrm{Nrf2}$ by interfering with recruitment of Nrf2 to the ARE, whereas nuclear levels of Nrf2 are not affected by ATRA ${ }^{10}$. In this study, the expression of Nrf2/ HO-1 in I/R group was decreased, but the expression of caspase-3 was increased, which is related to apoptosis ${ }^{30}$. Otherwise, the kidney injury scores and the blood indices in I/R group were worsen. These showed that ATRA indeed limited the expression of Nrf2, leading to increased apoptosis and aggravated kidney injury.

Taken together, ATRA impacts the kidney histopathology and function in renal ischemiareperfusion rat model by downregulating Nrf2 protein expression. However, in STZ-induced diabetic rats with renal ischemia-reperfusion injury, due to many renal protective factors of ATRA as above stated, the effect of inhibiting Nrf2 does not perform a central role. Conversely, accompanying with the expression of DJ-1 upregulated, Nrf2 protein expression also increased so that more Nrf2 exerted an anti-oxidant effect. The deficiency of this study is that it does not explore the relationship between ATRA and the DJ-1/Nrf2 pathway, and that the mechanism of ATRA renal protective in STZinduced diabetic rats with renal ischemia-reperfusion injury is still unclear.

\section{- Conclusion}

This study suggested that ATRA played a protective effect on renal ischemia-reperfusion injury in diabetic rats, which might relate to the DJ/Nrf2 pathway. Our results might provide a potential therapeutic strategy against diabetic renal ischemia-reperfusion injury.

\section{- Acknowledgement}

To Central Laboratory, Renmin Hospital of Wuhan University for their support of our study.

\section{- References}

1. Phillips AO, Baboolal K, Riley S, Grone H, Janssen U, Steadman R, Williams J, Floege J. Association of prolonged hyperglycemia with glomerular hypertrophy and renal basement membrane thickening in the Goto Kakizaki model of non-insulin-dependent diabetes mellitus. Am J Kidney Dis. 2001;37(2): 400-10. doi: 10.1053/ajkd.2001.21322. 
2. Hung CC, Lin HYH, Hwang DY, Kuo IC, Chiu YW, Lim LM, Hwang SJ, Chen HC. Diabetic retinopathy and clinical parameters favoring the presence of diabetic nephropathy could predict renal outcome in patients with diabetic kidney disease. Sci Rep-Uk. 2017;7. doi: 10.1038/s41598017-01204-6.

3. Wang Y, Zhong YJ, Wang YY, Xing J, Wang ZM. All-trans retinoic acid prevents the development of type 1 diabetes by affecting the levels of interferon gamma and interleukin 4 in streptozotocin-induced murine diabetes model. Genet Mol Res. 2016, 15(1). doi: 10.4238/ gmr.15017522.

4. Eltony SA, Elmottaleb NA, Gomaa AM, Anwar MM, El-Metwally TH. Effect of all-trans retinoic acid on the pancreas of streptozotocin-induced diabetic rat. Anat Rec (Hoboken). 2016;299(3):334-51. doi: 10.1002/ar.23307.

5. Kim CS, Park JS, Ahn CW, Kim KR. All-trans retinoic acid has a potential therapeutic role for diabetic nephropathy. Yonsei Med J. 2015;56(6):1597-603. doi: 10.3349/ ymj.2015.56.6.1597.

6. Sun $Q$, Shen ZY, Meng $Q T$, Liu HZ, Duan WN, Xia ZY. The role of DJ-1/Nrf2 pathway in the pathogenesis of diabetic nephropathy in rats. Ren Fail. 2016;38(2):294-304. doi: 10.3109/0886022X.2015.1120119.

7. No JH, Kim YB, Song YS. Targeting nrf2 signaling to combat chemoresistance. J Cancer Prev. 2014;19(2):111-7. doi: 10.15430/JCP.2014.19.2.111.

8. Arnold P, Mojumder D, Detoledo J, Lucius R, Wilms H. Pathophysiological processes in multiple sclerosis focus on nuclear factor erythroid-2-related factor 2 and emerging pathways. Clin Pharmacol. 2014;6:35-42. doi: 10.2147/ CPAA.S35033.

9. Zhang $Y$, Rong $S$, Feng $Y$, Zhao L, Hong J, Wang R, Yuan W. Simvastatin attenuates renal ischemia/reperfusion injury from oxidative stress via targeting Nrf2/HO-1 pathway. Exp Ther Med. 2017;14(5):4460-6. doi: 10.3892/ etm.2017.5023.

10. Wang XJ, Hayes JD, Henderson CJ, Wolf CR. Identification of retinoic acid as an inhibitor of transcription factor Nrf2 through activation of retinoic acid receptor alpha. Proc Natl Acad Sci U.S.A. 2007;104(49):19589-94. doi: 10.1073/ pnas.0709483104.

11. Wang WW, Sun AL, Lv W, Cheng J, Lv SS, Liu XC, Guan GJ, Liu G. TRB3, up-regulated in kidneys of rats with type1 diabetes, mediates extracellular matrix accumulation in vivo and in vitro. Diabetes Res Clin Pract. 2014;106(1):101-9. doi: 10.1016/j.diabres.2014.07.015.

12. Tong F, Tang X, Li X, Xia W, Liu D. The effect of insulinloaded linear poly(ethylene glycol)-brush-like poly(Ilysine) block copolymer on renal ischemia/reperfusioninduced lung injury through downregulating hypoxia-inducible factor. Int J Nanomedicine. 2016;11:1717-30. doi: 10.2147/IJN.S99890.

13. McWhinnie DL, Thompson JF, Taylor HM, Chapman JR, Bolton EM, Carter NP, Wood RF, Morris PJ. Morphometric analysis of cellular infiltration assessed by monoclonal antibody labeling in sequential human renal allograft biopsies. Transplantation. 1986;42(4):352-8. doi: 10.1097/00007890-198610000-00004.
14. Hu B, Wu YH, Tong F, Liu J, Shen XH, Shen RL, Xu GT. Apocynin alleviates renal ischemia/reperfusion injury through regulating the level of zinc and metallothionen. Biol Trace Elem Res. 2017;178(1):71-8. doi: 10.1007/ s12011-016-0904-z.

15. Xu G, Gu H, Hu B, Tong F, Liu D, Yu X, Zheng Y, Gu J. PEGb-(PELG-g-PLL) nanoparticles as TNF-alpha nanocarriers: potential cerebral ischemia/reperfusion injury therapeutic applications. Int J Nanomedicine. 2017;12:2243-54. doi: $10.2147 /$ IJN.S130842.

16. Dechow C, Morath C, Peters J, Lehrke I, Waldherr R, Haxsen $V$, Ritz E, Wagner J. Effects of all-trans retinoic acid on reninangiotensin system in rats with experimental nephritis. Am J Physiol Renal Physiol. 2001;281(5):F909-19. doi: 10.1152/ ajprenal.2001.281.5.F909.

17. Han SY, So GA, Jee YH, Han KH, Kang YS, Kim HK, Kang SW, Han DS, Han JY, Cha DR. Effect of retinoic acid in experimental diabetic nephropathy. Immunol Cell Biol. 2004;82(6):56876. doi: 10.1111/j.1440-1711.2004.01287.x.

18. Schaier M, Liebler S, Schade K, Shimizu F, Kawachi $H$, Grone HJ, Chandraratna R, Ritz E, Wagner J. Retinoic acid receptor alpha and retinoid $\mathrm{X}$ receptor specific agonists reduce renal injury in established chronic glomerulonephritis of the rat. J Mol Med. 2004;82(2):11625. doi: 10.1007/s00109-003-0510-3.

19. Nishimura C, Kuriyama K. Alteration of lipid peroxide and endogenous antioxidant contents in retina of streptozotocin-induced diabetic rats: effect of vitamin A administration. Jpn J Pharmacol. 1985;37(4):365-72. doi: 10.1254/jjp.37.365.

20. Sifuentes-Franco S, Pacheco-Moises FP, RodriguezCarrizalez AD, Miranda-Diaz AG. The role of oxidative stress, mitochondrial function, and autophagy in diabetic polyneuropathy. J Diabetes Res. 2017;2017:1673081. doi: 10.1155/2017/1673081.

21. Babizhayev MA, Strokov IA, Nosikov VV, Savel'yeva EL, Sitnikov VF, Yegorov YE, Lankin VZ. The role of oxidative stress in diabetic neuropathy: generation of free radical species in the glycation reaction and gene polymorphisms encoding antioxidant enzymes to genetic susceptibility to diabetic neuropathy in population of type $\mathrm{i}$ diabetic patients. Cell Biochem Biophys. 2015;71(3):1425-43. doi: 10.1007/s12013-014-0365-y.

22. Dehdashtian E, Mehrzadi S, Yousefi B, Hosseinzadeh A, Reiter RJ, Safa M, Ghaznavi H, Naseripour M. Diabetic retinopathy pathogenesis and the ameliorating effects of melatonin; involvement of autophagy, inflammation and oxidative stress. Life Sci. 2018;193:20-33. doi: 10.1016/j. Ifs.2017.12.001.

23. Cui W, Min X, XuX, Du B, Luo P. Role of Nuclearfactorerythroid 2-related factor 2 in diabetic nephropathy. J Diabetes Res. 2017;2017:3797802. doi: 10.1155/2017/3797802.

24. Shokeir AA, Hussein AM, Barakat N, Abdelaziz A, Elgarba M, Awadalla $A$. Activation of nuclear factor erythroid 2-related factor 2 (Nrf2) and Nrf-2-dependent genes by ischaemic pre-conditioning and post-conditioning: new adaptive endogenous protective responses against renal ischaemia/ reperfusion injury. Acta Physiol (Oxf). 2014;210(2):342-53. doi: 10.1111/apha.12164. 
25. Choucry MA, Khalil MNA, El Awdan SA. Protective action of Crateva nurvala Buch. Ham extracts against renal ischaemia reperfusion injury in rats via antioxidant and antiinflammatory activities. J Ethnopharmacol. 2017;214:47-57. doi: 10.1016/j.jep.2017.11.034.

26. Ma Q. Role of nrf2 in oxidative stress and toxicity. Annu Rev Pharmacol Toxicol. 2013;53:401-26. doi: 10.1146/annurevpharmtox-011112-140320.

27. Saito H. Toxico-pharmacological perspective of the Nrf2Keap1 defense system against oxidative stress in kidney diseases. Biochem Pharmacol. 2013;85(7):865-72. doi: 10.1016/j.bcp.2013.01.006.
28. Kobayashi M, Yamamoto M: Molecular mechanisms activating the Nrf2-Keap1 pathway of antioxidant gene regulation. Antioxid Redox Signal. 2005;7(3-4):385-94. doi: 10.1089/ars.2005.7.385.

29. Osburn WO, Kensler TW. Nrf2 signaling: An adaptive response pathway for protection against environmental toxic insults. Mutat Res. 2008;659(1-2):31-9. doi: 10.1016/j.mrrev.2007.11.006.

30. Lee MW, Hirai I, Wang HG. Caspase-3-mediated cleavage of Rad9 during apoptosis. Oncogene. 2003;22(41):6340-6. doi: $10.1038 /$ sj.onc.1206729.

\section{Correspondence:}

Xia Zhongyuan

Phone: 15701292818

xiazhongyu-an2005@aliyun.com

Received: Sept 03, 2019

Reviewed: Nov 08, 2019

Accepted: Dec 06, 2019

\section{Conflict of interest: none}

Financial sources: National Natural Science Foundation of China ( $n \circ 81501648$ ) and Natural Science Foundation of Hubei Province (no 2019CFB670)

${ }^{1}$ Research performed at Department of Anesthesiology, People's Hospital of Wuhan University, Wuhan, Hubei, China. 